\title{
The development of scientific reasoning of preschool children: Micro-analysis of mind-material-body integration
}

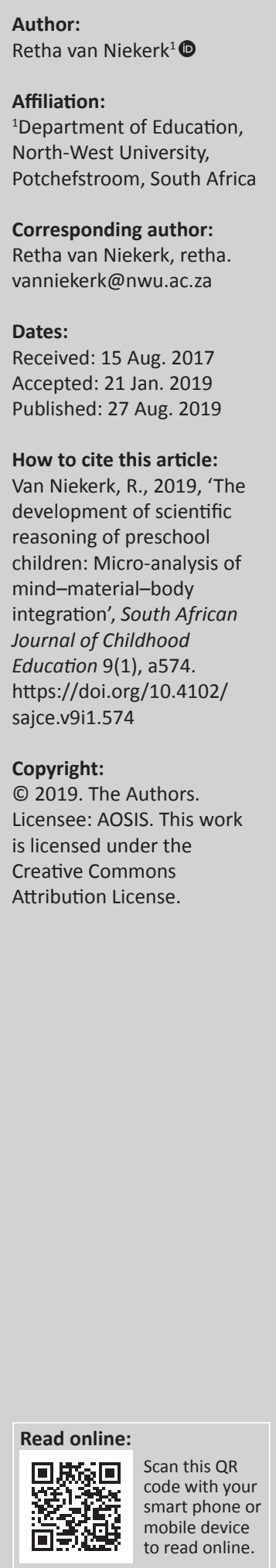

Background: This article argues that the emergence of scientific reasoning in the preschool years could be augmented by caregivers and preschool teachers through nurturing 'Habits of Mind' (HOM) and 'Habits of Body' (HOB) of young children. This type of mind-materialbody integration is proposed from an epistemological position that comprises a Hybrid (morphinuum) of theories about early learning and human development.

Aim: The aim of this article is to present an exemplar of the capacity of one preschooler to show emergence and integration of two HOM, namely conjecturing and reasoning with invariance, in tandem with the Habit of Body (HOB), namely hand-eye coordination that can lay the foundation for scientific reasoning in the early years.

Setting: The study referred to in this article is an exemplar (case study) taken from a larger, 18-month educational design research intervention, the 'Little African Scientists Project'. That study investigated the emergent scientific HOM and HOB through a multimodal material approach to pedagogy at preschool level (Grade RR to R).

Methods: A three-layered digital video analysis was utilised to interpret the data pertaining to a specific interaction of one child, who was manipulating magnets during one of the many free-play activities that formed part of the larger project.

Results: Several specific HOM and $\mathrm{HOB}$ were evident in the one child who was engaged in an activity in which he was moving magnets. Two HOM that emerged were those of making and testing conjectures and reasoning with invariance, while the HOB that emerged was a close alignment of hand movement and eye coordination.

Conclusion: This type of close observation and micro-analysis could be utilised for studies of more children in similar settings.

Keywords: Emergence; Conjecturing; Habits of Mind (HOM); Habits of Body (HOB); Image; Invariant.

\section{Introduction}

\section{Mind-material-body integration}

This study was designed to inquire into the development of scientific reasoning of preschoolers from the theoretical perspective of integrating 'Habits of Mind' (HOM) and 'Habits of Body' (HOB) through specific material interactions in a preschool classroom setting.

This notion of integration of body and mind dates back to antiquity and bridges cultures and time. Tahiri (2016) mentions that the Arabic-Islamic scholar Ibn Sīnā (born in 980 CE/370 AH), known in the west as Avicenna, stated that:

Conceptualising the intelligibles is acquired only through the intermediary of sensory perception in one way, namely that sensory perception takes the perceptible forms and presents them to the imaginative faculty, and so those forms become subjects of our speculative intellect's activity, and thus there are numerous forms there taken from the perceptible humans. (p. 49)

The idea of morphing mind and body, as a more accurate reflection of the sense-making mode of humans, has also been adopted by many mathematics and science educators. Radford (2009) points to the highly specialised interaction between language, and auditory, tactile and kinaesthetic sensory 'channels'. Nemirovsky and Ferrara (2009) are of the opinion that all language utterances are accompanied by a range of multimodal expressions including facial expression, 
gesture, tone of voice, sound production, eye motion, body poise, gaze and so forth. De Villiers (2003) points to the fact that in the domain of mathematics, it is well known that experimentation, which includes physical measurement and handling of materials, forms the cornerstone of many mathematical discoveries. This experimentation is in many cases done merely on the basis of intuition and conjecturing. In other words, the ability to make a conjecture about a phenomenon and then proceed to prove or disprove it is a basic HOM that is found in the toolbox of most mathematicians, as well as many scientists in general. Closely linked to experimentation and/or conjecturing is the HOM called invariant reasoning, which is the awareness that a scientist needs to have about the relationship between what stays the same and what changes during experimentation.

Schmandt-Besserat and Erard (2008) point to the possible relationship that existed between material and cognition related to how number emerged out of clay in Neolithic times. This resonates with counting phases and corresponds with the material embodiment of different types of counters, as expressed by young learners, in their initial development of counting in the process of number concept development. Ihde and Malafouris (2018) express the opinion that humantechnology relations are not representational relations but embodiment relations. It is further their contention that the medium per se takes on an active role of mediation that is not static and linear but contributes to the idea of plasticity of the brain in learning. So, the mediator is not necessarily only another human being, but also the material medium as such.

In his groundbreaking work, 'How things shape the mind', Malafouris (2013:3) begins by asking the question 'Where does the mind stop and the rest of the world begin?'. This very question is the one that frames the event represented in this research. This mind-material-body integration approach that is used as a lens to describe this research therefore takes cognisance of the complexity of teaching and learning in the 21st century and utilises a theoretical approach that would best be described as being a hybrid of theories. Such theories would include an approach of 'morphing', whereby a number of theories, such as discursive psychology (Roth 2008), multimodality (Kress 2010), mediation or media (Ruckriem 2010), materiality (Grassby 2005), activity and expansiveness (Sannino \& Engeström 2017), systems (Bronfenbrenner 1979), semiotics (Arzarello 2006), HOM (Cuoco, Goldenberg \& Mark 1996), design (Goswami 2015; Selander 2008), neuropsychological (Johnson \& De Haan 2015; Panksepp 2011) and material engagement theory (Malafouris 2010), are combined in what is called a dynamic, contracting and expanding educational morphinuum of theories.

\section{Physics and young children}

Foundational work was done by Piaget (1977), regarding the interaction between young learners and their physical environment. He proposed four criteria that need to be met when very young learners engage in physics activities which involve movements, namely, that children should be able to (1) produce by themselves the actions that lead to the movements, (2) bring about the change in the actions by themselves, (3) perceive the change to be immediate and (4) experience the change in movement visually. Piaget (1954) also stated that, therefore, children should not be exposed to work with magnets because the nature of the force is invisible. In other words, the force involved in magnetism is not produced by the learners themselves, which is in direct violation with one of the previously mentioned criteria.

Despite this caution by Piaget, quite a large volume of work has been carried out in recent years by researchers who investigated young children and their explorations with magnets (Ashbrook 2005; Barrow 2000; Christidou et al. 2009; Constantinou et al. 2013; Kalogiannakis, et al. 2017; Van Hook \& Huziak-Clark 2007). From the literature regarding the interaction of preschoolers with magnets, two main types of inquiries emerged, namely (1) which types of forces are exhibited, referring to attraction and/or repulsion, and (2) what types of materials react to these attraction and/or repulsion forces (Kalogiannakis et al. 2017). What is absent from the majority of the research investigations involving preschool children is another very important aspect of magnetic force, namely the very idea of the magnitude of the force itself. This, initially, seems to be a far-fetched idea when thinking about the capacity of preschoolers, seeing that in the majority of cases, they do not yet fully comprehend whole numbers. This article, to the contrary, reports on just such a voluntary exploration of a preschooler investigating the nature of the magnetic force, per se, between two magnets.

An important feature of a preschooler's development, alongside that of language, is the triadic developmental aspect of movement-action-activity, as originally coined by Rubinshtein (1946). This triad of movement-action-activity implies that very young children need to develop perceptual skills that are learnt through their interaction with objects in their everyday exploitations, which includes motor systems and biomechanical as well as other kinds of reactions formed during early life. The diversities of perceptual skills concerning interactions of the body with the external environment can be grouped under the umbrella term 'proprioception', which was introduced in 1906 by Charles Scott Sherrington (Kaya, Yertutanol \& Calik 2018:3). Proprioception is multifaceted and does not only provide us with knowledge of the position and spatial orientation of our limbs but also gives information concerning their movement and the ways in which we are able to determine the force we exert as we interact with objects and, conversely, the force that objects exert on us (Blundell 2008).

\section{The work of Zaporozhets and compatriots}

Zaporozhets (2002) argued that the contribution that Vygotsky's theory of the systemic structure of human perception has made is still relevant. Extensive studies (Arievitch \& Haenen 2005; Leontiev 2005; Zaporozhets 1965, 2002d; Zinchenko, Pruzhinin \& Shchedrina 2011) by Soviet 
researchers that can be related to the work of Vygotsky have shown that the effector components of perceptual activities that deal with the interrelated associations of the hands and eyes are crucial components of this movement-actionactivity triad in the early years of development.

Of specific interest to this research is the work done by Alexander Zaporozhets (1965) and his compatriots related to the domain of the development of sensory processes of the preschool child. Their extensive focus on the role of the hand and the eye in relation to object manipulation gives us entrance into the very nature of this mind-material-body relationship exhibited by very young children. Zaporozhets and Lukov (2003:30-46), under the supervision of A.N. Leontiev, conducted experiments in the context of floating and sinking, while investigating scientific reasoning capacities in children between the ages of 3 and 7 years. Although these experiments were done in a specific context (floating and sinking), the findings are of relevance as generalisations that can be extrapolated to other contexts. These generalised extrapolations are relevant in the sense that they deal with the very important aspect of the type of reasoning and the aspects that affect it, which are characteristics of children at preschool age, irrespective of the specific content they are involved in. These tendencies include:

- the way in which an object reacts, as presented in consecutive tasks, affects the reasoning of the learner

- the actions of the learner himself or herself on the object determine the way in which the learner makes judgements about the properties of the object

- the nature of the objects themselves, as reflected in everyday activities, that is experienced independent of the actual investigation, affects reasoning about cause and effect

- the nature of the activities that the learner engages in changes as the learner gains more experience with objects in richer and more complex contexts, which ultimately affects his or her reasoning

- a reasoning style, particularly associated with this age phase, linked to hand-eye coordination

- the sequence in which preschoolers experience phenomena (task sequence), either naturally through their own explorations or through teacher-mediated tasks, affects their reasoning about scientific phenomena.

From the above-mentioned tendencies, two distinct types of judgements form the defining criteria for making decisions about the properties of how objects react, namely forecasting judgement and affirmative judgement (Zaporozhets \& Lukov 2002:36, 37). In other words, the way in which a child reasons about objects and their reactions is influenced by the nature of the relationship the child builds as he or she interacts with the objects both physically and mentally. In general, forecasting judgements are made based on the interaction with previous objects and the reasoning related to them. This implies that memory and the ability to recall previous events all play a role, while affirmative judgements are made based on the facts in front of the child. From the work of Zaporozhets and
Lukov (2002:37), it can be concluded that there is a constant interplay between these two types of judgements, and as the child gains more experience with a situation, the two types of judgements come closer to each other as not being opposites in their final analysis of a problem.

All these developments in the preschool years require the mastery of cultural tools (Veraksa 2011:79) that are different for different age groups and even for learners of the same age groups but coming from diverse cultural backgrounds. So, the challenge is up to the researchers who engage with preschoolers to, firstly, be aware of these differences and, secondly, to identify the tools relevant to the development of perceptual actions for a specific group they are investigating.

\section{Research design and methods Setting}

I participated in an institutionally funded and ethically cleared 18-month research project. The research was conducted in a farm school in the North West province of South Africa, with learners starting in Grade RR (two years before formal schooling) in June 2014 and completing Grade R in November 2015. Thirty-six learners participated, from their Grade RR year through to their Grade $R$ year. Their starting ages were between 48 and 54 months. All the learners spoke Sesotho as their first language and they were taught in both English and Sesotho during their school day. During their Grade RR year, their teacher was an English first language speaker assisted by a support teacher, who was a Sesotho first language teacher. During their Grade R year, the children were taught by a teacher who had Sesotho as her first language, but with a very good command of English. The medium of instruction during both these years was a combination of English and Sesotho.

The primary aim of the study was to identify, through an educational design research approach (Plomp \& Nieveen 2013), a teaching-learning trajectory (Van den HeuvelPanhuizen 2003) for teaching sciences in the reception year at preschool level. An important secondary aim was to focus on how English featured in their learning (Kress 2005, 2013) and how the language was used by the children specifically as representations of scientific HOM.

Although this article reflects on the activities of only one child, the contribution that it makes adds to the idea expressed by Cobb, Confrey, diSessa, Lehrer and Schauble (2003) regarding educational design research. They are of the opinion that there exists a very close relationship between the development of theory and the improvement of instructional design in order to bring about understanding of new forms of learning. This implies that all forms of learning (formal, informal, incidental, etc.) are utilised and considered, in a retrospective way, to add to theory building and vice versa. This said, it is important to caution that one of the important limitations to this study is the fact that the researcher does not understand the home language of the learners and, in this specific activity (event), did not intervene and ask any questions in order to clarify certain actions of the learner. 
The exemplar that is discussed in this article is an activity (event) that one learner (approximately 5 years and 10 months old) engaged in during a free-play session 6 months into the project. In this activity, the entire group of 36 learners were playing in smaller groups, using manipulatives that had been used during the past 6 months. No indication was given to the learners as to how to use any of the manipulatives. Some of the manipulatives were small wooden blocks, paper clips and plastic containers. At this stage, the only exposure that the learners had had with this specific tool (magnets) was a short game, introduced by the researcher in a previous contact session, of investigating how one can push and pull these magnets across a table in a 'racing game fashion'.

The following section is a description of the unfolding of the event utilising a digital video analysis approach and represents but one way of interpreting this event.

\section{Method of data capturing and analysis}

The method of data capturing and analysis that was used in the research can be described as a digital video analysis that consists of three layers of analysis, namely a common sense interpretation, a situated practice interpretation and a thematic interpretation (Fleer 2013:28-31). Although all three data analysis levels are discussed separately in this article, they occur concurrently in practice to enable the researcher to contribute to the iterative nature of the educational design research protocol. It therefore implies that the reader should take notice of the emergent interpretations within and across the three different representation levels.

\section{Common sense interpretation}

The recorded video, taken by the researcher, was digitally analysed against the overall background of what transpired during the entire free-play activity - in this case, the activities of all the children interacting together in an open space, as a free-play activity was considered. The series of figures (Figure 1 and Figure 2) are an example of such a trajectory.

Figure 1 and Figure 2 show a voluntary participation of different learners in a relaxed and dynamic way. Most of the learners are not staying with one object for too long but they explore the different objects in their own time. Although many of the learners are working in close proximity, there is little verbal communication.

\section{Situated practice interpretation}

The digital analysis of raw data involves describing min or sec by min or sec analysis of what is transpiring when the magnets are being manipulated by the one learner. The sequence (Figure 1 to Figure 9) represents the progression of the approximate $8 \mathrm{~min}$ and $35 \mathrm{~s}$ of voluntary, consciously regulated movement (Zaporozhets 2002:67), that the learner engaged in. The series of events is subdivided into four phases, namely (1) investigating the push or pull (force) phenomena, (2) investigating the relationship between the push force while the size or weight (magnitude) of one of the magnets is changed, (3) investigating the relationship between the push force while the size or weight (magnitude) of both magnets is changed and (4) investigating the nature of only the push force as a function of the child's own actions (embodiment).

\section{Phase 1: Conjecturing about the push or pull (force) phenomenon}

Figure 3 shows how the learner is in the process of investigating the attraction and/or repulsion forces between the two differently coloured sides of the two magnets. The learner has established that like colours repulse and unlike colours attract. He selects the blue-on-blue pushing force to investigate the next activity. The entire activity focuses on the nature of the attraction and/or repulsion force as a function related to magnets' own coloured visual appearances, namely red and blue sides.

This sequence is the only time in the entire investigation that the learner also incorporates an action to check if the attraction forces are still in place. For the rest of the sequence (time), he does not again show the need to check if the attraction forces are still prevalent. He continues the rest of the investigations, utilising only the repulsion force. In this activity, it seems that there is a conjecturing about the nature of the forces, which is then checked empirically.

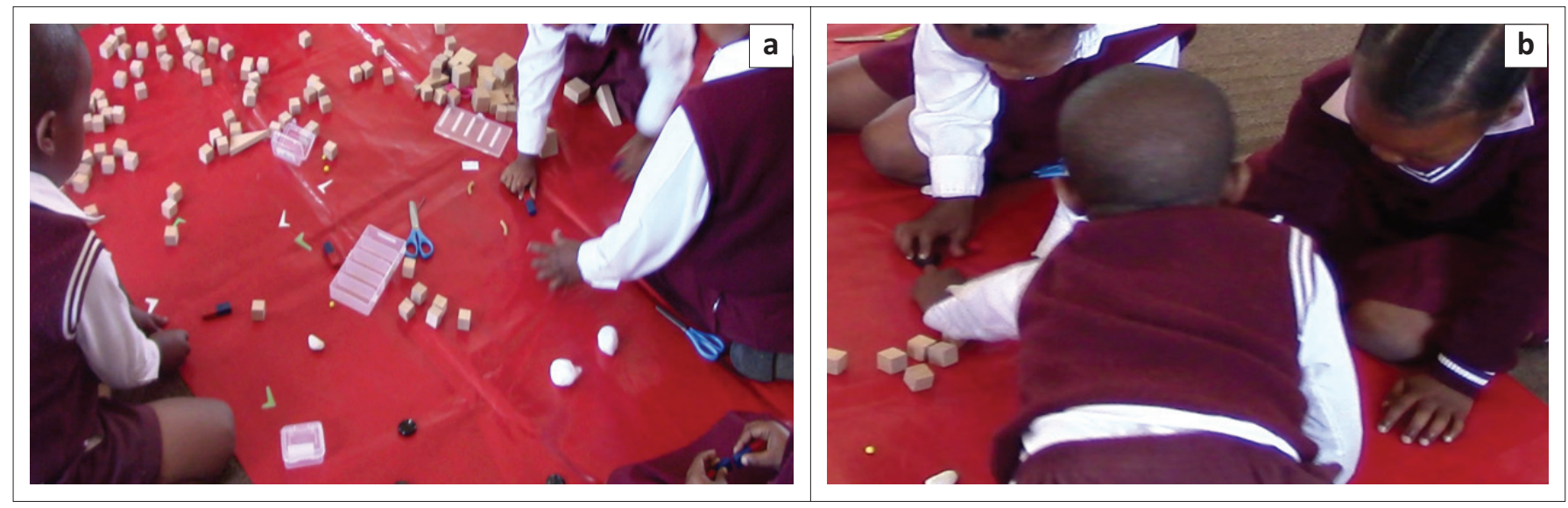

Source: (a \& b) Photograph taken by Dr Retha van Niekerk, taken in 2014, at Napo Combined Agricultural School, Bothaville, permission granted based on the clause of the ethics committee of the North-West University (ethical clearance number NWU-00188-14-S2)

FIGURE 1: (a) The learner is watching the other three learners pushing magnets away from one another. At this stage, the learner is not participating in any activity, but is just watching (28 seconds). (b) The learner is joining the others in trying to push away the magnets. They are all participating in the same activity ( 1 minute 9 seconds). 

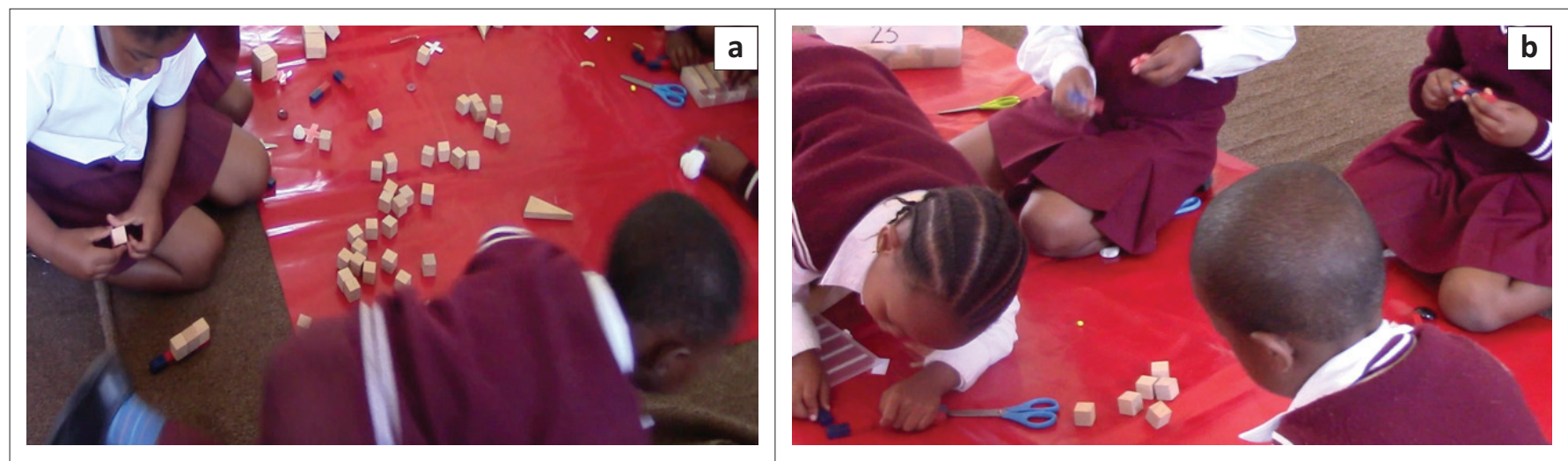

Source: (a \& b) Photograph taken by Dr Retha van Niekerk, taken in 2014, at Napo Combined Agricultural School, Bothaville, permission granted based on the clause of the ethics committee of the North-West University (ethical clearance number NWU-00188-14-S2)

FIGURE 2: (a) Everybody is reverting back to working on their own again. There is no sharing of the activity at this stage (2 minutes 1 seconds). (b) Another learner who was not part of the original group attempts to join this group, with resistance and partial ignorance from the group ( 2 minutes 59 seconds).

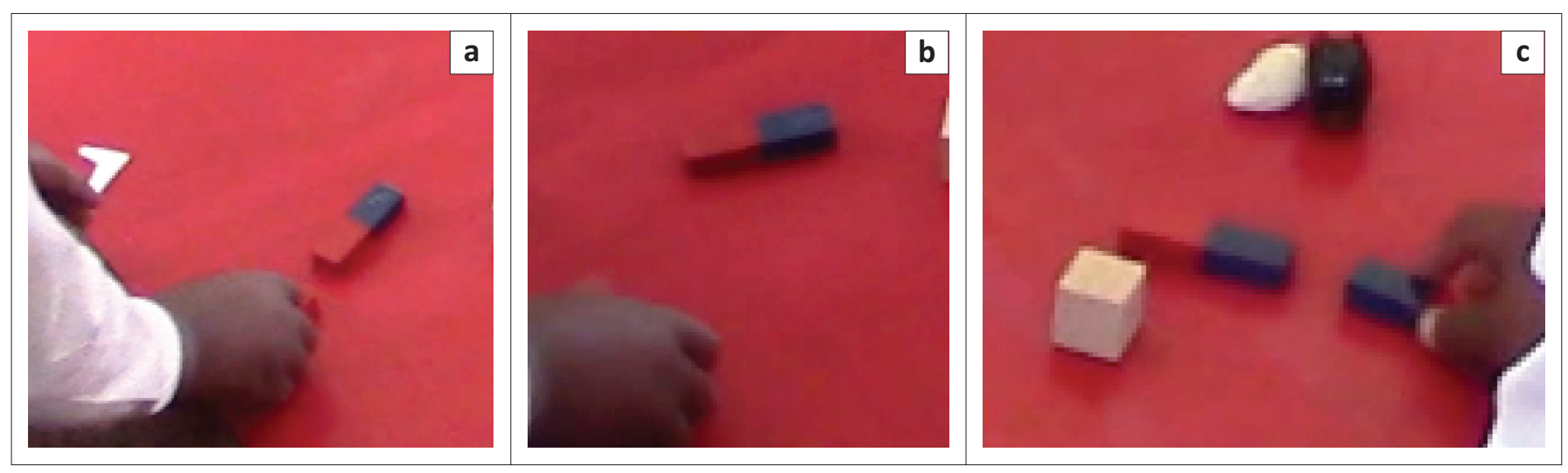

Source: (a, b \& c) Photograph taken by Dr Retha van Niekerk, taken in 2014, at Napo Combined Agricultural School, Bothaville, permission granted based on the clause of the ethics committee of the North-West University (ethical clearance number NWU-00188-14-S2)

FIGURE 3: (a) Pushing the red part of the receiving magnet with the red part of the controlled magnet (repulsion occurs between red parts) (24 seconds). (b) Changing the orientation of the controlled magnet to blue as pushing part and attracting the red part of the receiving magnet (attraction occurs between red and blue parts) (29 seconds). (c) Keeping the controlled magnet the blue as the pushing part, but changing the receiving magnet to blue as the receiving part (repulsion occurs between blue parts) ( 2 minutes 12 seconds).
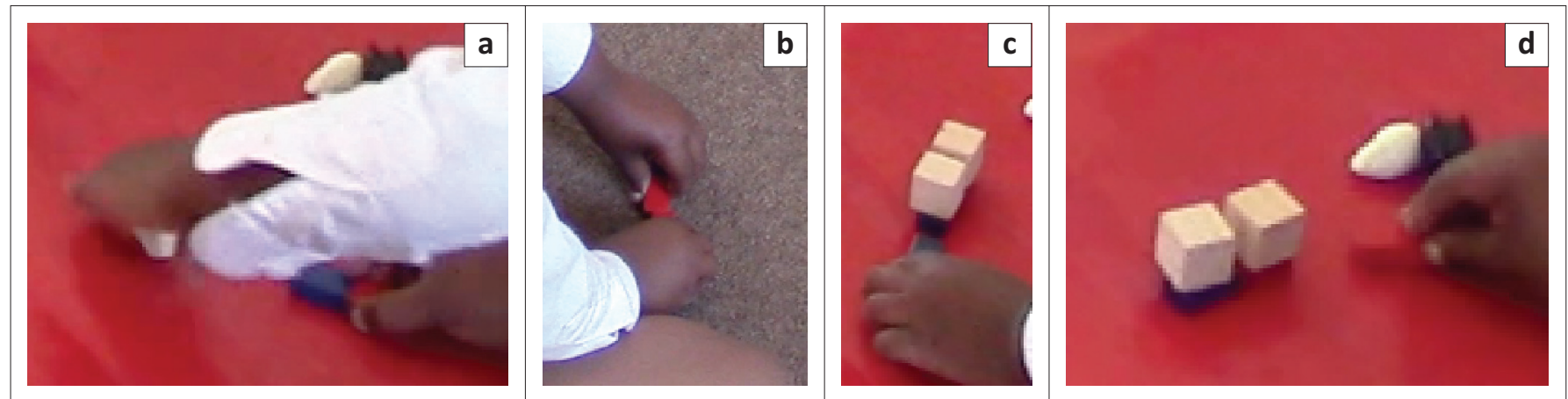

Source: (a, b, c \& d) Photograph taken by Dr Retha van Niekerk, taken in 2014, at Napo Combined Agricultural School, Bothaville, permission granted based on the clause of the ethics committee of the North-West University (ethical clearance number NWU-00188-14-S2)

FIGURE 4: (a) Continuing using the blue-on-blue pushing part and loads one block on the receiving magnet ( 2 min 18 seconds). (b) Checking to make sure that the blueon-blue pushing force is able to still push when two blocks are loaded ( 2 minutes 21 seconds). (c) Proceeding to load a second block on the receiving magnet ( 2 minutes 27 seconds). (d) Checking if the magnets can be pushed by changing to a red-on-red pushing part again ( 2 minutes 33 seconds).

\section{Phase 2: Investigating the relationship between the push force while only the size or weight (magnitude) of one of the magnets is changed}

Phase 2a: Introducing two blocks: In Figure 4, it can be seen that the learner continues using the blue-on-blue repulsion force, while he is loading the receiving magnet with two blocks, before he checks the repulsion force. In Figure 4d he also checks to see if the red-on-red repulsion force is still able to move the loaded (with two blocks) receiving magnet. Here he changes the rest of the investigation by using the red-onred pushing force.

This entire sequence focuses on two things, namely introducing the first variable, while verifying the ability to 

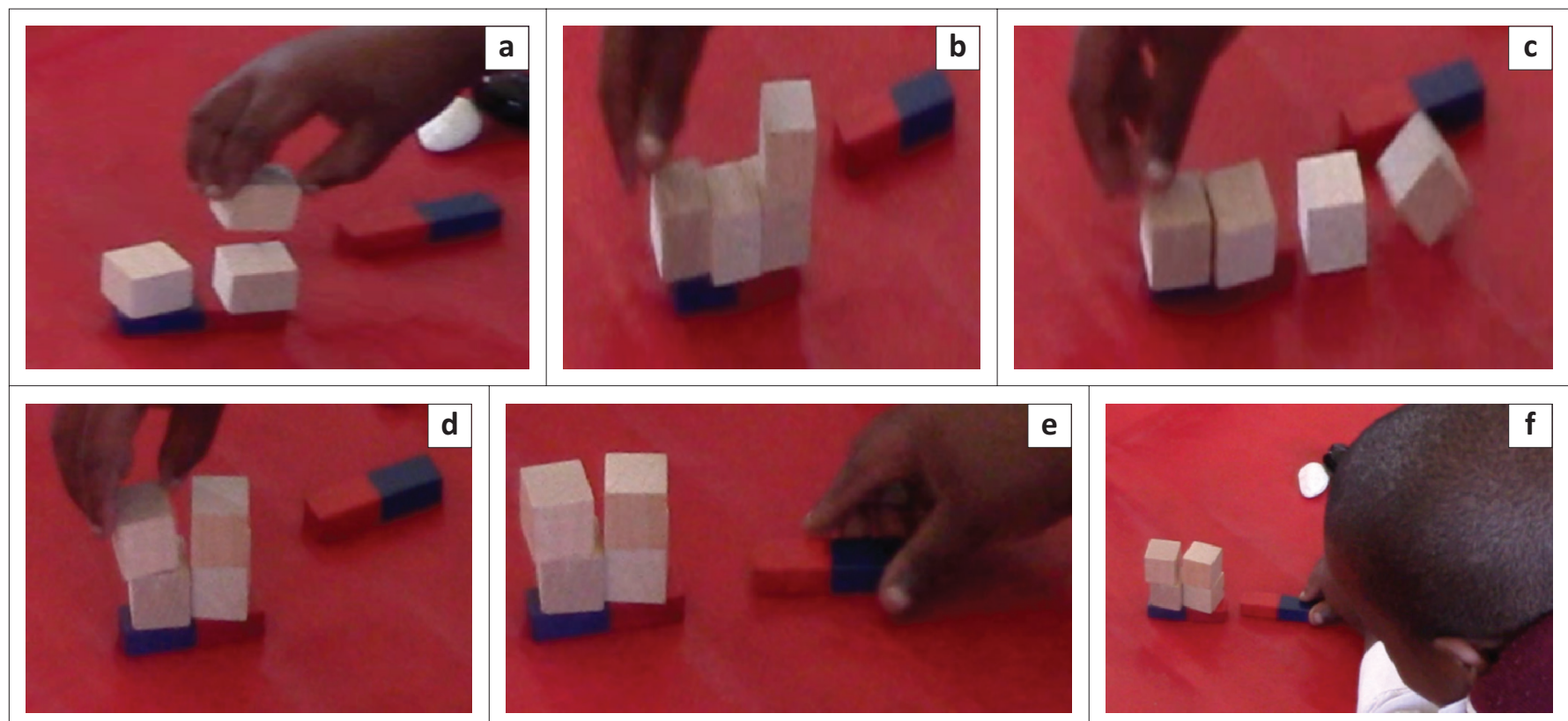

Source: (a, b, c, d, e \& f) Photograph taken by Dr Retha van Niekerk, taken in 2014, at Napo Combined Agricultural School, Bothaville, permission granted based on the clause of the ethics committee of the North-West University (ethical clearance number NWU-00188-14-S2)

FIGURE 5: (a) Satisfied that the red-on-red force works well, continuing to load a third block onto the receiving magnet ( 2 minutes 49 seconds). (b) Without testing any push (force) yet, the learner proceeds to put a fourth block on the blue part of the receiving magnet, for which he has already made space ( 2 minutes 55 seconds). (c) Unfortunately, the space on the magnet for accommodating the blocks is not enough and the entire structure of blocks collapses onto the floor next to the magnet ( 2 minutes 56 seconds). (d) When the learner rectifies this, he now stacks it in a different orientation to the previous one, seeing that the previous alignment made the structure collapse ( 3 minutes 4 seconds). (e) The learner proceeds to try and push this new structure containing the four blocks ( 3 minutes 5 seconds). (f) He makes quite an effort to push the entire new structure to the point where he changes posture and goes about this task, trying different orientations of the pushing magnet to the receiving magnet ( 3 minutes 10 seconds).

move the receiving magnet, even though there is a change in magnitude. He is also making sure that the initial discovery, namely that like sides repulse one another, has not changed.

Phase 2b: Introducing blocks three and four: Figure 5 shows a sequence of six figures that reflects how the learner continues to use the red-on-red pushing force, while loading a third and a fourth block on the receiving magnet (Figure $5 \mathrm{a}-\mathrm{c}$ ). Unfortunately, this configuration of four blocks is not a stable construction. He is still not testing the pushing force of the loaded four-block structure, but he is changing the orientation of the four blocks to make a more stable construction, so that they do not collapse, as is shown in Figure 5c. Once he is satisfied that the new configuration of four blocks is stable, he spends some time pushing the receiving magnet, making sure it can be pushed without collapsing (Figure 5e and f).

The prominent feature of this phase is the fact that there is a deliberate intent to make the receiving magnet's magnitude as big as possible, before checking to see if it can still be moved. This is an empirical verification step in the process of optimising the magnitude of the receiving magnet.

Phase 2c: Introducing blocks five to seven: Figure 6 shows the learner continuing the investigation of the pushing force on the receiving magnet. After he is satisfied that the fourblock structure can be pushed, he adds a fifth block to the receiving magnet. He continues to add a sixth and a seventh block, without checking the pushing force (Figure 6a-c).
In his attempt to load the seventh block, he struggles because the structure keeps collapsing. He is eventually distracted by another learner close by (Figure 6d and Figure 6e). This phase is characterised by the fact that he is now not testing the pushing force after adding just one or two more blocks.

This phase is a continuation of the previous phase, but now the learner seems to be looking at the maximum number of blocks that can be stacked onto the receiving magnet, before he tests the pushing force.

\section{Phase 3: Investigating the relationship between the push force while the size or weight (magnitude) of both magnets is changed}

Phase 3a: Introducing three blocks: Figure 7, in this phase, introduces a change in focus. After the initial distraction, while not being able to keep a structure of seven blocks on the receiving magnet stable, he checks the pushing force of red-on-red once again, as in Figure 4d of Phase 2a (Figure 7a). He initially starts to reload the controlling magnet with one block, but then changes his task by stacking the receiving magnet only, while immediately checking the pushing capacity with only one block on the receiving magnet (Figure $7 \mathrm{~b}$ and $\mathrm{c}$ ). He eventually proceeds to load two more blocks simultaneously on the receiving magnet, without checking the pushing force (Figure $7 \mathrm{~d}$ and e).

Two features that emerge from this phase include, firstly, checking that the pushing force of the red-on-red magnets is still in place. Then, secondly, he initially attempts to load a block on the pushing magnet, but decides against that and proceeds to load the receiving magnet. The next 

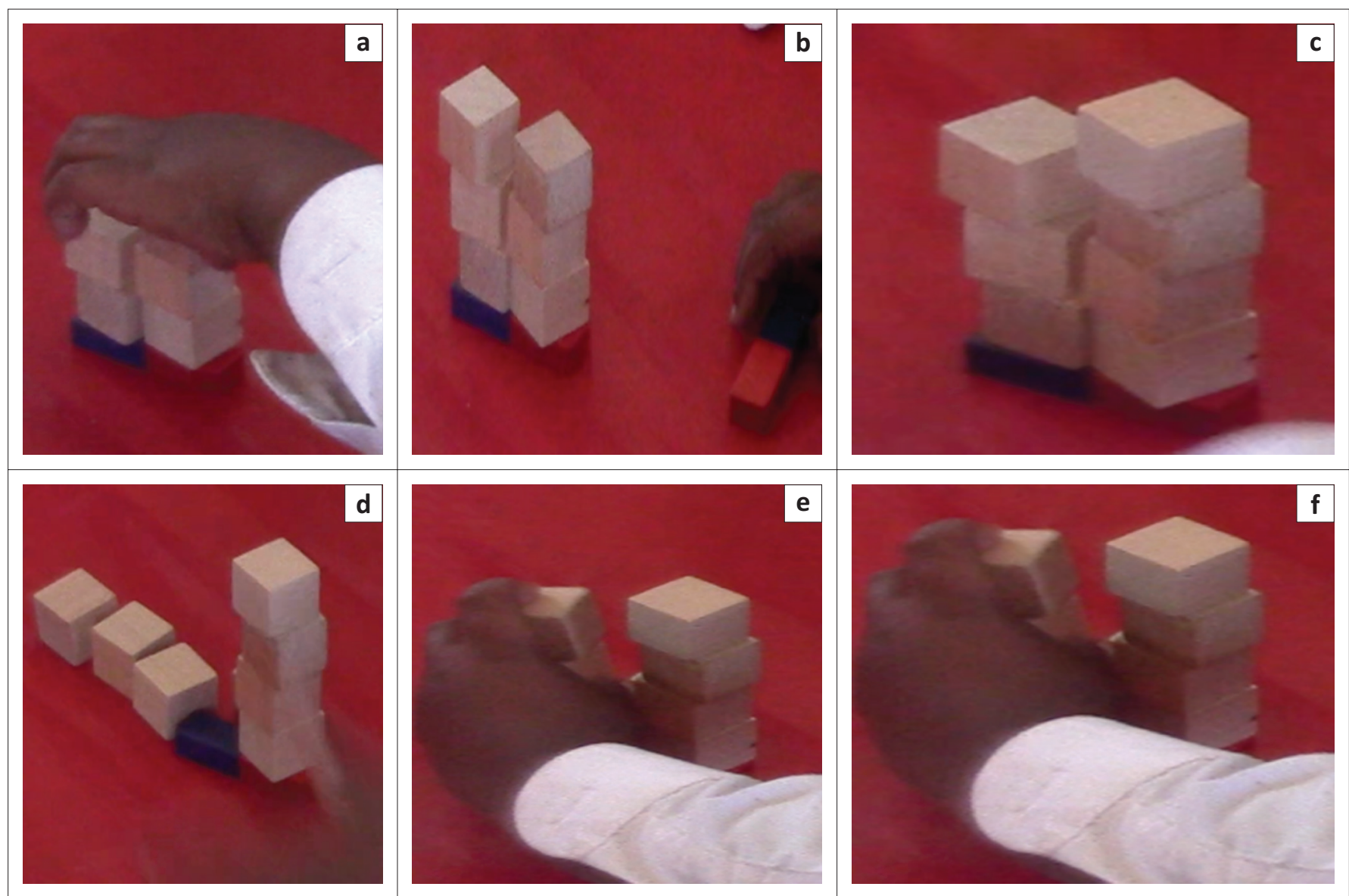

Source: (a, b, c, d, e \& f) Photograph taken by Dr Retha van Niekerk, taken in 2014, at Napo Combined Agricultural School, Bothaville, permission granted based on the clause of the ethics committee of the North-West University (ethical clearance number NWU-00188-14-S2)

FIGURE 6: (a) Satisfied that the four-block structure can be moved, he then moves to place a fifth block on the receiving magnet ( 3 minutes 15 seconds). (b) Without checking to see if it can be moved, he adds a sixth block ( 3 minutes 17 seconds). (c) He immediately continues, without checking the pushing force, to add a seventh block ( 3 minutes 21 seconds). (d) The structure collapses ( 3 minutes 25 seconds). (e) He immediately attempts to get the structure up again, but fails ( 3 minutes 30 seconds). (f) Although he puts in quite an effort to get the structure up again, he has lost some concentration, while he also gets distracted by another learner close by, who is attempting to use some of the blocks that he has originally used to 'load' the magnets ( 3 minutes 40 seconds). (At this stage, although his eyes moved away from his construction, he kept on fiddling with the blocks in his hands, turning it over and over).

step in Phase $3 b$ extends the initial intent of loading the controlling magnet as well.

Phase 3b: Equalising the number of blocks on both magnets: Figure 8 depicts the completion of the sequence, which reflects a change in focus. This is a new investigation that involves a second change in the invariant reasoning HOM. He is not changing the magnitude of the receiving magnet anymore, but he is now changing the magnitude of the controlling magnet as well. He first adds one block onto the controlling magnet (Figure 8a); he then continues to load a second and a third block onto the controlling magnet, without checking the pushing force (Figure $8 b-d$ ). Once both magnets have the same number of blocks and the same configuration, he attempts to push the magnets (Figure 8e).

There is a clear indication here of an intentional change in the magnitude of both the magnets towards an equivalent status. This activity takes him some time, because he has to continuously stop to fix the structures on both magnets, because they are collapsing. He also gets distracted by some learners close by. Although he attempts to push these equally loaded magnets together, at no stage do the magnets touch each other in this pushing activity.

\section{Phase 4: Investigating the nature of only the push force as a function of the learner's own actions}

Figure 9 introduces an entirely new focus, away from the magnets as sole force production, towards the use of the whole body. He has discarded the idea of checking the pushing forces with both magnets being loaded with blocks and reverts to trying to push the magnets together, using his own two hands.

So, the initial receiving magnets in the previous phases that were not controlled by his hands are now controlled by both his hands (Figure 9). He spends a considerable amount of time trying to push the two magnets' red parts together by varying the position of his hands, but also utilising the stability of the floor (Figure 9a and d). He moves between the air (Figure $9 \mathrm{~b}$ and c) and the floor in trying to stabilise the movement of the magnets. He eventually succeeds in pushing the two magnets together on the floor (Figure 9d). At this stage, the period was ended by the teacher. (He did not stop of his own accord.) 


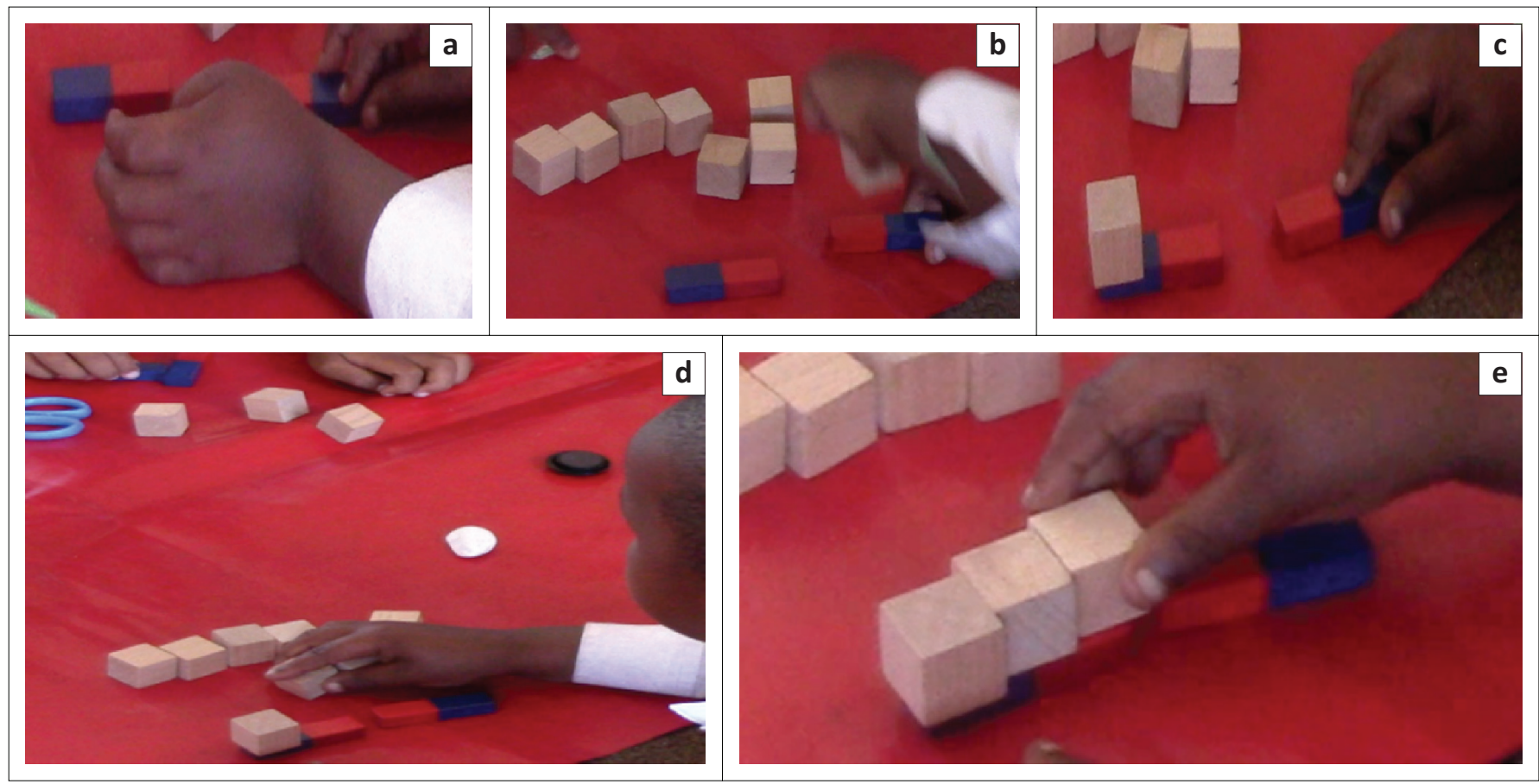

Source: (a, b, c, d \& e) Photograph taken by Dr Retha van Niekerk, taken in 2014, at Napo Combined Agricultural School, Bothaville, permission granted based on the clause of the ethics committee of the North-West University (ethical clearance number NWU-00188-14-S2)

FIGURE 7: (a) He abandons the blocks and checks the initial pushing force of the two magnets (red-on-red) ( 3 minutes 54 seconds). (b) He starts stacking the blocks again, but he now stacks them onto the controlling, pushing magnet first ( 3 minutes 58 seconds). (c) He suddenly changes his mind and reverts back to stacking blocks onto the receiving magnet. He then tests the pushing capacity by trying several times to see if it works with blocks on the magnet ( 4 minutes 3 seconds). (d) He pauses and his gaze wanders because his attention is momentarily distracted by another learner doing some pushing activities in sight. He already has his hand on two more blocks that he wants to add ( 4 minutes 14 seconds). (e) After a moment of distraction, he proceeds to add the two additional blocks onto the receiving magnet ( 4 minutes 17 seconds).
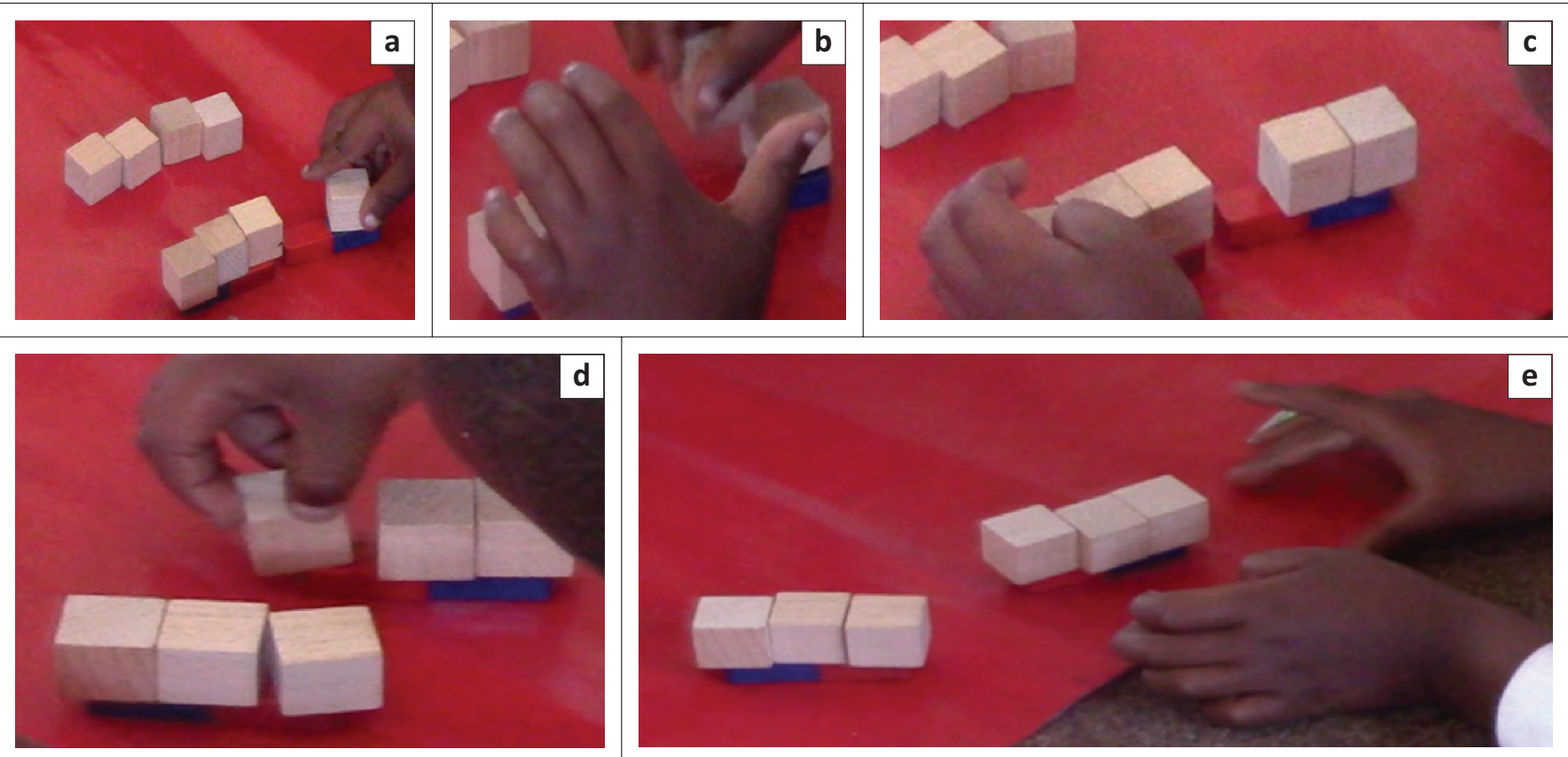

Source: (a, b, c, d \& e) Photograph taken by Dr Retha van Niekerk, taken in 2014, at Napo Combined Agricultural School, Bothaville, permission granted based on the clause of the ethics committee of the North-West University (ethical clearance number NWU-00188-14-S2)

FIGURE 8: (a) He then adds the next block (fourth), not onto the receiving magnet, like the activities reflected in phase 2, but onto the controlling, pushing magnet (4 minutes 19 seconds). (b) He adds the fifth block onto the controlling magnet. He takes quite a lot of care to make sure that the blocks do not collapse (4 minutes 22 seconds). (It can also be seen in the time that elapses between completing the two final products.) (c) He takes some time to look at this structure without doing much. $\mathrm{He}$ also starts looking around at what the others are doing (it is the first time that he is breaking his focus from his own activity). Some time elapses with distractions (4 minutes 35 seconds). (d) He goes one step further by loading a third block, something that was most probably his intention from the start to make the number of blocks on each magnet the same ( 5 minutes 16 seconds). (e) He now tries to push the magnets that are loaded with the same number of blocks on each magnet (5 minutes 40 seconds). After this activity, he gets distracted by other learners close by, after he has struggled for some time to push the two magnets, while he has to continuously struggle to keep the blocks stacked on top of the two magnets when they are being pushed. 


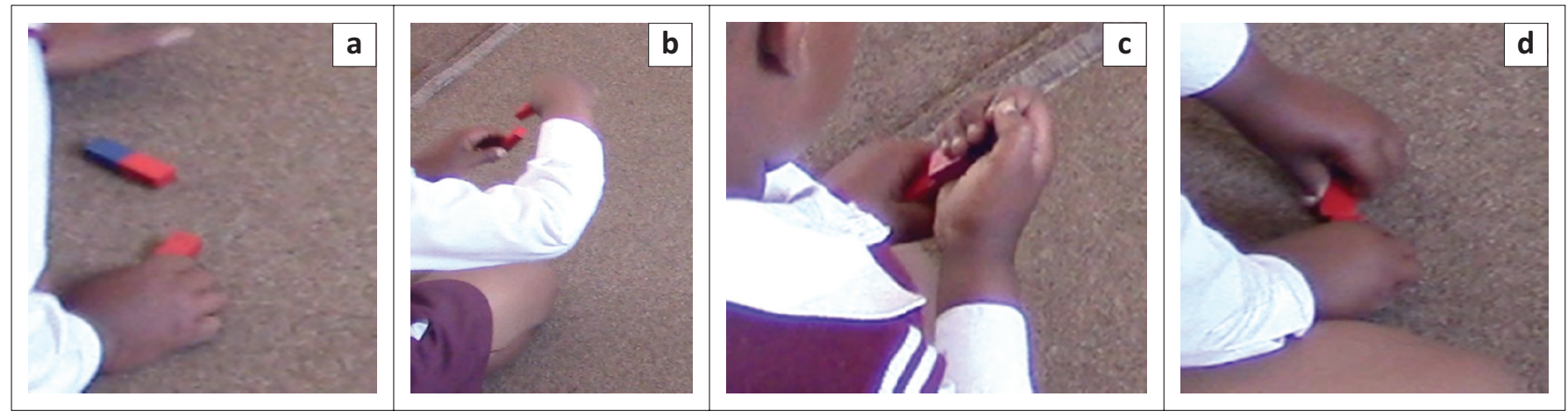

Source: (a, b, c \& d) Photograph taken by Dr Retha van Niekerk, taken in 2014, at Napo Combined Agricultural School, Bothaville, permission granted based on the clause of the ethics committee of the North-West University (ethical clearance number NWU-00188-14-S2)

FIGURE 9: (a) He is temporarily distracted by other learners who are trying to pack some wooden blocks into plastic containers, but soon returns to his magnets. Here he is again trying to push the red parts together (7 minutes 55 seconds). (b) He is now determined to push the two magnets' red components together. He lifts it up from the carpet and tries to do so in the air (7 minutes 59 seconds). (It seems, once again, that he is interested in the force that is at play.) (c) He spends quite a lot of time trying to push the two red parts together in the air with his hands ( 9 minutes 26 seconds). (d) After quite some time, he finally manages to push the two red parts together with his hands (10 minutes5 seconds).

It could be that the learner does not manage to adapt the conditions of his own knowledge that are required for the conditions of the task in order to solve it, but proceeds to adapt the conditions of the task per se in order to solve it. This is sometimes a classical way in which many learners who do not yet have enough initial knowledge operate - they change the conditions of the problem to be solved.

\section{Thematic interpretation}

The third and final level of the digital video analysis goes beyond the separate video files and seeks to integrate the and practice that emerged in a non-linear fashion, while aiming to capture change. Three main themes form the focus in order to illuminate aspects of the research question stated on the outset, namely explicating the nature of the interrelated collaboration between mind, material and body, while utilising a multimodal instructional approach to scientific reasoning at preschool level.

\section{Emergent scientific habits of mind}

Conjecturing: The ability to make a 'thought experiment', also called a conjecture, is the starting foundation for any scientific experimentation (De Villiers 2003). It implies that the experimenter (learner) must have a question that interests him and it further implies that there should be scope for investigating the questioned phenomena.

It seems that the fact that the magnets were repulsing one another in such a way that it was not easy to push them together by hand was of great interest, not only to the learner but also to the group that he was part of. The fact that there was a range of materials (e.g. magnets, small blocks, paper clips and plastic containers) available to utilise in the exploration seemed to assist in the investigation. This learner is going through a process of empirical checking and changing conditions which is indicative of the process of conjecturing. The fact that he is not perturbed by sporadic failure because of blocks falling off the magnets, or other learners competing for his attention, is indicative of this.
Although the learner never verbally explained the purpose with his experimentation, it was apparent at the end of the investigation (Phase 4) that the magnitude of the 'force' that keeps repulsing the magnets was a question that he wanted to answer.

Invariance of magnitude: Chaille and Britain (2003) sound a word of caution regarding the number of variables that is introduced into any experimentation with young learners. They are cautioning that the number of variables is dependent on the age and relative development of a child related to a specific content domain. The one main variable that this learner dealt with during his experimentation was the deliberate changing of the magnitude aspects of the magnets' repulsion 'force', in a non-random way. This is a reflection of an emergent idea of reasoning with invariants. The main invariant that is changed from the start is the size and/or weight of the magnets and its relation to overcoming the repulsion 'force'. He ends the activity (Phase 3b) by discarding the magnets' manipulated forces, as a causal variable, and utilises his own 'force' through different attempts at pushing the magnets together with his hands.

\section{Material as mediator}

The material nature of magnets lends itself to a range of possibilities when encountered in diverse contexts. This learner participated in a task designed through his own initiative. By implication, it means that the questions posed and ensuing investigation did not come from any direct mediating source in the form of a teacher or the researcher. There is a strong possibility that the previous experience that he had with the magnets, in a play context, led to this interest. The fact that the magnets moved in different ways based on the positions relative to one another also seemed to interest him. For example, the sideways pushing/pulling was different to the head-on pushing and pulling. The very movement of the magnets that occurred as the magnitude was changed clearly played some mediating role in his entire investigation. The use of the blocks as objects to change the 
weight (magnitude) of the magnets also adds to the idea that the availability of material and their affordance in this case of being able to be stacked contributed to the entire invariance exploration.

\section{Emergent habits of body}

The learner's use of his hands in this activity is deliberate and prominent. The hands are clearly supported by the eyes as well as the rest of the body in the overall determination of the magnitude and effect of the pushing forces on the magnets. The instances when his eyes and his hands are not working in tandem are characterised by staring into open space, while his hands are still holding the magnets. As the process continues, it is clear that there is a refinement in the way he uses his hands to manipulate the different orientations of the magnets.

\section{Ethical considerations}

All the research was covered by ethical clearance that was granted by North-West University (Ethical clearance number: NWU-00188-14-A2).

\section{Conclusion and recommendations}

If we would want all learners to be involved in authentic activities in the context of the sciences, one of the challenging aspects would be how to design and mediate a teaching and learning trajectory that allows for participation that is driven, to a large extent, by the enquiry of the learners themselves. That implies that the learners would need to be allowed to ask the questions. For authentic scientific investigations, it would imply that although initially the questioning should or could come from the teacher as mediator, it could gradually be supplanted and become the sole domain of the learner, assisted by the materials they are interacting with.

In other words, the role of the mediator or teacher should not necessarily be replaced but changed. It would imply that the teacher would now be challenged to come up with supporting the investigation and taking on the role of facilitator. One way in which this could have emerged, if there had been an opportunity for it in the mentioned exemplar reported in this article, would have been for the researcher to ask questions such as, 'how can you determine, or what is the size or magnitude of the push or pull (force magnitude) in another way, but not using wooden blocks?' 'How can you determine what is the influence of distance on this pushing force?' 'What is the direction in which the push or pull is biggest or smallest?' Unfortunately, the researcher did not intervene at all, as it was a free-play activity.

The conclusion can be made that in order to accommodate the diversity of skills and background of the preschool child in the 21st century, great care will have to be taken when decisions are made concerning the teaching and learning of science-related content. Instead of agonising about which content to teach and prematurely trying to contrive contexts, caregivers, teachers and researchers could benefit more by focusing on the development of specific HOM and HOB and their interrelationship to the available materials in a specific context. Once it is agreed that very young learners are capable of developing quite sophisticated HOM, such as conjecturing, reasoning with invariance and visualising, in conjunction with very important $\mathrm{HOB}$, the onus will be on teachers to capitalise on any event that learners are exploring, whether it is in a playful or in a more structured, enquiry-based environment.

Ultimately, the challenge to anyone involved with teaching and learning of preschoolers regarding scientific reasoning and exploration would be to acquaint himself or herself with the range of $\mathrm{HOM}$ and $\mathrm{HOB}$ that are intuitively being expressed by learners in an emergent format within specific contexts, as they indulge in a variety of playful as well as teacher-directed (inquiry-based) activities, dependent on their specific age phase, cultural context and material surroundings. The implication of this is that the caregiver or teacher should be able to link the external manifestation of a specific HOM or HOB to an appropriate set of developmental milestones that are ultimately supported by relevant theories of teaching and learning.

This approach of utilising mind-material-body integration as lens through which to view teaching and learning the sciences in the 21st century deals with two important issues simultaneously. Firstly, it addresses the problematic question of uniformalisation, through a rigidly prescribed curriculum, of the content, didactics (teaching method) and contexts suitable for sciences teaching and learning at preschool level. Secondly, it moves the focus of all caregivers, teachers and researchers involved, to the very important aspect of making the situated child in context their focus when dealing with development of scientific reasoning. Consequently, it could eliminate the false prescriptions and impact of over-ambitious or under-ambitious viewpoints, perpetuated by people not familiar with the complexities of the unique teaching and learning environment in every school, while still ensuring equality and excellence in the development of science-related activities for preschoolers.

The very real challenge, therefore, will be to the caregivers and teachers, who should need to possess the necessary knowledge and skills to be able to identify and support this emergent mind-material-body phenomenon, while simultaneously considering the specific age and total cultural context of their learners. This implies that instead of making the content or the methodology (didactics) the driving principle in the design of a science curriculum for preschoolers, the mind-materialbody idea becomes the guiding framework. This ultimately puts the responsibility back in the court of teacher and caregiver trainers, to make sure that this domain of knowledge is properly disseminated at both preservice and in-service education facilities. 
Finally, a cautionary note to all researchers and educators at the forefront of all research as stated by Giesecke (2005) is that what we need in this post-typographical era is a revised understanding of communication, knowledge acquisition and information processing. This ultimately implies that theories of teaching and learning related to the sciences will have to be revisited and revised through processes of theory morphing and co-construction, aiming for the emergence of new theories applicable to teaching and learning the sciences during the preschool years, in the 21 st century.

\section{Acknowledgements Competing interests}

The author declares that they has no financial or personal relationships that may have inappropriately influenced them in writing this article.

\section{Author's contributions}

R.v.N. was the sole contributor to this article.

\section{Funding Information}

This research received no specific grant from any funding agency in the public, commercial, or not-for-profit sectors.

\section{Data availability statement}

Data sharing is not applicable to this article as no new data were created or analysed in this study.

\section{Disclaimer}

The views and opinions expressed in this article are those of the author and do not necessarily reflect the official policy or position of any affiliated agency of the author.

\section{References}

Arievitch, I.M. \& Haenen, J.P., 2005, 'Connecting sociocultural theory and educational practice: Galperin's approach', Educational Psychologist 40(3), 155-165. https:// doi.org/10.1207/s15326985ep4003_2

Arzarello, F., 2006, 'Semiosis as a multimodal process', Revista Latinoamericana de Investigación en Matemática Educativa RELIME 9(Extraordinario 1), 267-299.

Ashbrook, P., 2005, 'More than messing around with magnets', Science and Children 43(2), 20-23.

Barrow, L.H., 2000, 'Do elementary science methods textbooks facilitate the understanding of magnet concepts?', Journal of Science Education and Technology 9(3), 199-205. https://doi.org/10.1023/A:100948743

Blundell, B.G., 2008, An introduction to computer graphics and creative 3-D environments, Springer Science \& Business Media, London.

Bronfenbrenner, U., 1979, The ecology of human development, Harvard University Press, Cambridge, MA and London.

Chaille, C. \& Britain, L., 2003, The young child as scientist. A constructivist approach to science education, Pearson Education, Inc., San Francisco.

Christidou, V., Kazela, K., Kakana, D. \& Valakosta, M., 2009, 'Teaching magnetic attraction to preschool children: A comparison of different approaches', International Journal of Learning 16(2), 115-128.

Cobb, P., Confrey, J., DiSessa, A., Lehrer, R. \& Schauble, L., 2003, 'Design experiments in educational research', Educational Researcher 32(1), 9-13. https://doi. org/10.3102/0013189X032001009

Constantinou, C., Raftopoulos, A., Spanoudes, G. \& Natsopoulos, D., 2013, 'Preschoolers' construction of operational definitions in magnetism', The Journal of Emergent Science 5, 6-21.
Cuoco, A.A., Goldenberg, E.P. \& Mark, J., 1996, 'Habits of mind: An organizing principle for mathematics curriculum', Journal of Mathematical Behavior 15, 375-402. https://doi.org/10.1016/S0732-3123(96)90023-1

Cuoco, A., 2008, 'Mathematical habits of mind: An organizing principle for curriculum design', paper presented at a Project Next Session on Helping Students Develop Mathematical Habits on Mind, Joint Mathematics Meetings, San Diego, 8 January.

De Villiers, M., 2003, 'The value of experimentation in mathematics', paper presented at the 9th National Congress of AMESA, Cape Town, South Africa, June 30-04 July, 2003, pp. 174-185.

Fleer, M. \& Ridgway, A. eds., 2013, Visual methodologies and digital tools for researching with young children: Transforming visuality, vol. 10, Springer, Cham.

Giesecke, M., 2005, 'In search of post-typographical educational ideals', Die Entdeckung der kommunikativen Welt 1788, 481-510.

Goswami, U., 2015, Children's cognitive development and learning, Primary Review Trust, New York.

Grassby, R., 2005, 'Material culture and cultural history', Journal of Interdisciplinary History 35, 591-603.

Hodson, D., 2014, 'Nature of science in the science curriculum: Origin, development, implications and shifting emphases', in M.R Matthews (ed.), International handbook of research in history, philosophy and science teaching, pp. 911-970, Springer, Dordrecht.

Ihde, D. \& Malafouris, L., 2018, 'Homo faber revisited: Postphenomenology and material engagement theory', Philosophy \& Technology 1-20. Springer, Netherlands. https://doi.org/10.1007/s13347-018-0321-7

Johnson, M.H. \& De Haan, M., 2015, Developmental cognitive neuroscience. An introduction, The Atrium, Southern Gate, Chichester, West Sussex.

Kaya, D., Yertutanol, F.D.K. \& Calik, M., 2018, 'Neurophysiology and assessment of the proprioception', Proprioception in orthopaedics, sports medicine and rehabilitation, pp. 3-11, Springer, Cham.

Kalogiannakis, M., Nirgianaki, G.M. \& Papadakis, S., 2017, 'Teaching magnetism to preschool children: The effectiveness of picture story reading', Early Childhood Education Journal 46(5), 535-546. https://doi.org/10.1504/IJTCS.2018.090965

Kress, G.R., 2010, A socio semiotic approach to contemporary communication, Routledge, New York, N.Y.

Kress, G.R., 2005, 'Gains and losses: New forms of texts, knowledge, and learning', Computers and Composition 22, 5-22. https://doi.org/10.1016/j.compcom. 2004.12.004

Kress, G.R., 2013, 'Recognizing Learning: A perspective from a social semiotic theory of multimodality', in I. de Saint-Georges \& J.J. Weber (eds.), Multilingualism and multimodality current challenges for educational studies, pp. 119-131, Sense, multimodality
New York, N.Y.

Latash, M.L., Turvey, M.T. \& Bernshteĭn, N.A., 1996, Dexterity and its development, Lawrence Erlbaum, Mahwah, NJ.

Leontiev, A.A., 2006, 'Units and levels of activity', Journal of Russian \& East European Psychology 44(3), 30-46. https://doi.org/10.2753/RPO10610405440303

Leontiev, A.N., 2005, 'The genesis of activity', Journal of Russian \& East European Psychology 43(4), 58-71. https://doi.org/10.1080/10610405.2005.11059253

Malafouris, L., 2010, 'Metaplasticity and the human becoming: Principles of neuroarchaeology', Journal of Anthropological Sciences 88(4), 49-72.

Malafouris, L., 2013, How things shape the mind, MIT Press. Cambridge, MA and London.

Nemirovsky, R. \& Ferrara, F., 2009, 'Mathematical imagination and embodied cognition', Educational Studies in Mathematics 70(2), 159-174. https://doi. org/10.1007/s10649-008-9150-4

Panksepp, J., 2011, 'Antonio Damasio: Self comes to mind: Constructing the conscious brain. New York: Pantheon, 2010. ISBN 978-0-307-37875-0', Neuropsychoanalysis 13(2), 205-217.

Piaget, J., 1977, The development of thought: Equilibration of cognitive structures, transl. A. Rosin, Viking, Oxford.

Piaget, J., 1954, The child's construction of reality, Routledge, Miltonpark.

Plomp, T. \& Nieveen, N., 2013, Educational design research - Part B: Illustrative cases, SLO, Enschede, Netherlands.

Radford, L., 2009, 'Why do gestures matter? Sensuous cognition and the palpability of mathematical meanings', Educational Studies in Mathematics 70(2), 111-126. mathematical meanings', Educational Studies
https://doi.org/10.1007/s10649-008-9127-3

Roth, W.M., 2008, 'The nature of scientific conceptions: A discursive psychological perspective', Educational Research Review 3(1), 30-50.

Rubinshtein, S.L., 1946, Foundations of general psychology, Academic Science, Moscow.

Rückriem, G., 2009, 'Digital technology and mediation: A challenge to activity theory', in A. Sannino, H. Daniels \& K.D. Gutiérrez (eds.), Learning and expanding with activity theory, pp. 88-111, Cambridge University Press, Cambridge.

Ryle, G., 1976/1949, The concept of mind, Penguin, Harmondsworth, Middlesex.

Sannino, A. \& Engeström, Y., 2017, 'Studies of expansive learning: Foundations, findings and future challenges', in H. Daniels (ed.), Introduction to Vygotsky, pp. 100-146, Routledge, London.

Schmandt-Besserat, D. \& Erard, M., 2008, 'Origins and forms of writing', in Handbook of research on writing: History, society, school, individual, text, pp. 7-22, Lawrence Erlbaum Associates, New York, N.Y. 
Selander, S., 2008, 'Designs of learning and the formation and transformation of knowledge in an era of globalization', Studies in Philosophy and Education 27(4), 267-281. https://doi.org/10.1007/s11217-007-9068-9

Tahiri, H., 2016, Mathematics and the mind: An introduction into Ibn Sinā's theory of knowledge, Springer, Cham.

Van den Heuvel-Panhuizen, M., 2003, 'Guides for didactical decision making in primary school mathematics education: The focus on the content domain of estimation Skriftserie for Nasjonalt Senter for Matematikki Opplaeringen 1, 139-152.

Van Hook, S.J. \& Huziak-Clark, T.L., 2007, 'Tip-to-tail: Developing a conceptual model of magnetism with kindergartners using inquiry-based instruction', Journal of Elementary Science Education 19(2), 45-58.

Veraksa, N.E., 2011, 'Development of cognitive capacities in preschool age', International Journal of Early Years Education 19(1), 79-87. https://doi.org/10. 1080/09669760.2011.571003

Vygotsky, L.S. \& Cole, M., 1978, 'LS Vygotsky', in M. Cole, V. John-Steiner, Scribner and Souberman (eds.), Mind in society, Harvard University Press, Cambridge, MA and London.
Vygotsky, L.S., 1999, The collected works of L S Vygotsky, volume 6, scientific legacy, transl. R.W. Rieber, Kluwer Academic Publishers, New York, N.Y.

Zaporozhets, A.V., 2002, 'Perception, movement, and action', Journal of Russian \& East European Psychology 40(4), 53-93. https://doi.org/10.2753/RPO10610405400453

Zaporozhets, A.V. \& Lukov, U.V., 2003, 'The development of reasoning in young children', Journal of Russian \& East European Psychology 40(4), 30-46. https:// doi.org/10.2753/RPO1061-0405400430

Zaporozhets, A.V., 1965, 'The development of perception in the preschool child', Monographs of the Society for Research in Child Development 30(2), 82-101. https://doi.org/10.1016/S0065-2407(08)60011-7

Zinchenko, V., Pruzhinin, B. \& Shchedrina, T.I., 2011, 'On a structural concept and levels of analysis of activity', Journal of Russian \& East European Psychology 49(4), 66-76. https://doi.org/10.2753/RPO1061-0405490404

Zuckerman, G.A., 2016, 'How do young schoolchildren learn to learn? The ability to learn: Defining concepts', Journal of Russian \& East European Psychology 53(1), 1-47. https://doi.org/10,1080/10610405.2016.1228779 